\title{
Intra- and Inter-Day Reliability of Body Composition Assessed by a Commercial Multifrequency Bioelectrical Impedance Meter
}

\section{(c) (1) $\circledast \odot$}

Authors

Laurent Bosquet ${ }^{1}$, Thomas Niort ${ }^{2}$, Maxime Poirault ${ }^{2}$

\author{
Affiliations \\ 1 Université de Poitiers, Faculté des sciences du sport, \\ Poitiers, France \\ 2 Universite de Poitiers, Sport sciences, Poitiers, France
}

\section{Key words}

hydration, fat mass, fat-free mass, relative reliability, absolute reliability

received 19.02 .2017

revised 27.04.2017

accepted 09.06.2017

\section{Bibliography}

DOI https://doi.org/10.1055/s-0043-113999

Sports Medicine International Open 2017; 1: E141-E146

(C) Georg Thieme Verlag KG Stuttgart · New York

ISSN 2367-1890

\section{Correspondence}

Prof. Laurent Bosquet

Université de Poitiers

Faculté des sciences du sport

8 allée Jean Monnet

86000 , Poitiers

France

Tel.: + 33/549/454 115, Fax: + 33/549/453 396

laurent.bosquet@univ-poitiers.fr

\begin{abstract}
The purpose of this study was to examine the intra- and interday reliability of body composition measurements provided by a commercial multifrequency bioelectrical impedance meter. Eighteen healthy, well-trained students in physical education from the same ethnic group were assessed on four consecutive days, both in the morning and in the evening. Indexes provided by the device were gathered in four categories: tissular, metabolic, hydric and ionic blocks. There was no systematic bias between repeated measures, regardless of time of day. Relative reliability was high to very high in the morning $(0.72<I C C<0.99)$ and moderate to very high in the afternoon $(0.61<I C C<0.99)$. Absolute reliability varied substantially between indexes $(1.5 \%<$ SEM $<15 \%)$. The minimum difference considered as real was proportionally altered, since it ranged from 4.2 to $41.5 \%$. In conclusion, body composition assessed with a multifrequency bioelectrical impedance meter requires a highly standardized protocol and adjusting the cut-off value for each parameter to ascertain during athlete follow-up that a real change has occurred. This assessment should preferably be scheduled in the morning in order to decrease these cut-off values.
\end{abstract}

\section{Introduction}

Body composition is generally considered as a determinant of performance in many sports, and as such is included in most of the testing procedures routinely used in the physiological assessment of athletes [2]. Among possible implications, it allows monitoring training-induced changes in order to formulate adapted nutritional and hydration recommendations, or to adjust the choice of training methods and the training load. It also allows monitoring the growth of children and adolescents by identifying those at risk of under- or over-fatness, and implementing a nutritional and/or a psychological follow-up when required [10].

Body composition covers different components that are not easy to assess simultaneously, including fat mass, fat-free mass, bone minerals and water content. Reference methods such as dual energy X-ray absorptiometry, hydrodensitometry or air-displacement plethysmography are expensive and often impractical in the follow-up of athletes when they are numerous. Anthropometric methods such as the sum of skinfolds, the body mass index and different circumference measurements are more accessible, but suffer important limitations. For instance, the sum of skinfolds is both operator-dependent and skinfold calliper-dependent, and relies on some questionable assumptions, such as a similar distribution of subcutaneous and internal fat in all individuals of the same sex [15]. The body mass index, which was developed to standardize weight by height, and other anthropometric measurements generally poorly reflect body composition $[5,16]$ and are particularly inadapted to sports where both body mass and muscular mass are important, such as rugby union.

Bioelectrical impedance is considered a good compromise between reference methods and anthropometric methods because 
it requires limited operator training, is not operator-dependent, does not expose subjects to ionizing radiation, is financially affordable, and is fast and easy to implement [14]. With this method, a low-level electrical current is passed through the body, and the resulting signal of impedance (or opposition to the flow of current) is used to assess the different components of body composition. In fact, considering that electrolytes in the body's water are excellent conductors of electrical current, the signal of impedance has the potential to provide a very good measure of total body water and fat-free mass (since water represents around $73 \%$ of this component). On the other hand, if we consider that adipose tissue has a low water content, it is a poor conductor of electrical current and can also be estimated from the signal of impedance. The main limitation with this method is the difficulty in converting resistance and reactance, the two components of the signal of impedance, into body composition [18]. It requires a regression equation developed from the comparison between this signal and body composition measured with a reference method. Considering that the signal of impedance may differ according to the analyzer $[8,19]$ or individual characteristics such as ethnic group, sex, age and physical fitness [14], these equations are complex to develop. They also represent the real added value of each commercial brand since they cannot be used interchangeably with other analyzers. Consequently, they require a specific examination of their reliability in order to determine the limits beyond which the changes observed during athlete follow-up were induced by the training intervention and not just due to measurement noise.

The aim of this study was therefore to examine the intra- and inter-day reliability of body composition measurements provided by a multifrequency bioelectrical impedance meter, the Z-Metrix (BioparHom, Bourget du Lac, France).

\section{Methods}

\section{Participants}

Eighteen healthy, well-trained students in physical education (13 males and 5 females) from the same ethnic group (white) gave their written informed consent to participate in the study. The usual training load of their academic program was 8 to $12 \mathrm{~h}$ of sport per week. Their mean \pm SD age, body mass and body height were $21.6 \pm 3.5$ years, $68.2 \pm 9.9 \mathrm{~kg}$ and $175.7 \pm 6.7 \mathrm{~cm}$, respectively $(22.1 \pm 3.9$ years, $72.2 \pm 7.8 \mathrm{~kg}$ and $178.8 \pm 4.8 \mathrm{~cm}$ for males and $20.4 \pm 2.1$ years, $57.8 \pm 6.8 \mathrm{~kg}$ and $167.4 \pm 2.3 \mathrm{~cm}$ for females). The protocol was conducted in accordance with recognized ethical standards and national/international laws [9]

\section{Experimental design}

Once included, participants came to a laboratory room of constant temperature $\left(21^{\circ} \mathrm{C}\right)$ on four consecutive days, both in the morning (between 10 and $12 \mathrm{AM}$ ) and in the evening (between 4 and $6 \mathrm{PM}$ ). This design was chosen because despite the care taken by staff to standardize tests and measurements used in the follow-up of athletes, it is not always easy to exactly control the time of day. Body mass was measured to the nearest $0.1 \mathrm{~kg}$ and body height to the nearest $0.5 \mathrm{~cm}$ at the beginning of each visit, with participants barefoot and wearing light clothes. Participants were asked to re- frain from strenuous training, to eat similarly and to abstain from alcoholic beverages $24 \mathrm{~h}$ before and throughout the 4 -day experimental period. They were also asked to arrive to the laboratory fully hydrated at least three hours after their last meal (i. e. breakfast or lunch) and to empty their bladder before each session.

\section{Body composition}

Skin was cleaned with alcohol before each measurement. Current injector electrodes with a surface size of $9 \mathrm{~cm}^{2}$ were placed just below the metacarpophalangeal joint in the middle of the dorsal side of the right hand, and just above the external malleolus of the right leg. Detector electrodes were placed on the dorsal side of the right wrist, midline with the radial styloid process and the ulnar styloid process, and on the right leg, $5 \mathrm{~cm}$ vertically above the upper side of the current injector electrode placed above the external malleolus.

According to the specifications of the manufacturer, participants adopted a standing position, with arms separated from trunk by about $30^{\circ}$ and legs separated by about $45^{\circ}$. Body composition was assessed with a Z-Metrix (BioparHom, Bourget du Lac, France). $Z$ Metrix is a multifrequency bioelectrical impedance meter that injects a low-intensity current $(77 \mu \mathrm{A})$ from the ankle to the wrist. Total body resistance, reactance and impedance are computed by using the frequency range of $1 \mathrm{kHz}$ to $1 \mathrm{MHz}$. Undisclosed equations validated by the manufacturer and equations available in the literature were used to estimate hydric, tissular, metabolic and ionic indicators presented in $>$ Table 1.

\section{Statistical analysis}

Standard statistical methods were used to calculate means and standard deviations. Normal Gaussian distribution of the data was verified by the Shapiro-Wilk test, and homoscedasticity by a modified Levene's Test.

Systematic bias, which refers to a general trend for measurements to differ in a particular direction between repeated tests [1], was assessed with a general linear model with repeated measures. The sphericity was checked by Mauchley's test. When the assumption of sphericity was not met, the significance of F-ratios was adjusted according to the Greenhouse-Geisser procedure when the epsilon correction factor was $<0.75$, or according to the HuynhFeldt procedure when the epsilon correction factor was $>0.75$. Multiple comparisons were made with the Newman-Keuls post hoc test.

Relative reliability, which represents the degree to which individuals maintain their position in a sample with repeated measurements [1], was assessed with the intraclass correlation coefficient (ICC model 2,1). Absolute reliability, which is the degree to which repeated measurements vary for individuals, was assessed with the standard error of measurement (SEM). Both the ICC and the SEM were computed from the breakdown of a two-way ANOVA (trials $x$ subjects) with repeated measures, as presented elsewhere [3]. We considered an ICC over 0.90 as very high, between 0.70 and 0.89 as high and between 0.50 and 0.69 as moderate [17]. Currier [4] has suggested that an ICC value higher than 0.80 was acceptable for clinical work. SEM can also be used to determine the minimum difference to be considered real (MD), which represents the limit under which the observed difference is within what we might ex- 
- Table 1 Origin of the equations used by the Z-Metrix to estimate body composition compartments.

\begin{tabular}{|c|c|}
\hline Parameter & Origin of equations \\
\hline \multicolumn{2}{|l|}{ Hydric indexes } \\
\hline Total water $(\mathrm{L})$ & $\begin{array}{l}\text { Undisclosed equation from } \\
\text { BioparHom }\end{array}$ \\
\hline Extracellular water $(\mathrm{L})$ & $\begin{array}{l}\text { Undisclosed equation from } \\
\text { BioparHom }\end{array}$ \\
\hline Intracellular water (L) & $\begin{array}{l}\text { Undisclosed equation from } \\
\text { BioparHom }\end{array}$ \\
\hline Extracellular/intracellular water ratio (\%) & Ratio \\
\hline Lean body mass hydration (\%) & $\begin{array}{l}\text { Undisclosed equation from } \\
\text { BioparHom }\end{array}$ \\
\hline \multicolumn{2}{|l|}{ Metabolic indexes } \\
\hline Metabolic activity (a.u.) & $\begin{array}{l}\text { Undisclosed equation from } \\
\text { BioparHom }\end{array}$ \\
\hline Quantity of active cells (\%) & Kotler et al. (1996) \\
\hline Availability of active cellular mass (a.u.) & $\begin{array}{l}\text { Barbosa et al. (2003); } \\
\text { Wells et al. (2004) }\end{array}$ \\
\hline Protein content of active cellular mass (\%) & Wang (2004) \\
\hline \multicolumn{2}{|l|}{ Tissular indexes a } \\
\hline Fat mass (\%) & $\begin{array}{l}\text { Undisclosed equation from } \\
\text { BioparHom }\end{array}$ \\
\hline Fat free mass (\%) & $\begin{array}{l}\text { Undisclosed equation from } \\
\text { BioparHom }\end{array}$ \\
\hline Bone mineral content (\%) & $\begin{array}{l}\text { Undisclosed equation from } \\
\text { BioparHom }\end{array}$ \\
\hline \multicolumn{2}{|l|}{ lonic indexes } \\
\hline Sodium (mmol) & Moore (1963) \\
\hline Potassium (mmol) & Moore (1963) \\
\hline Extracellular sodium (mmol. $\left.\right|^{-1}$ ) & Moore (1963) \\
\hline Extracellular potassium (mmol. $\mathrm{I}^{-1}$ ) & $\begin{array}{l}\text { Undisclosed equation from } \\
\text { BioparHom }\end{array}$ \\
\hline Intracellular potassium (mmol.I-1) & $\begin{array}{l}\text { Undisclosed equation from } \\
\text { BioparHom }\end{array}$ \\
\hline Creatinine $\left(\right.$ g.day $\left.^{-1}\right)$ & $\begin{array}{l}\text { Wang et al. (2003a and } \\
2003 b) ; \text { Wang (2004) }\end{array}$ \\
\hline Nitrogen (g) & James (1981) \\
\hline \multicolumn{2}{|l|}{ a.u.: arbitrary units } \\
\hline hese indexes are independent of each & \\
\hline
\end{tabular}

pect to see in repeated testing just due to measurement noise [21]. It is calculated as follows:

$\mathrm{MD}=\mathrm{SEM} \times 1.96 \times \sqrt{2}$

Statistical significance was set at $\mathrm{p}<0.05$ level for all analyses. All calculations were made with Statistica 6.0 (Statsofts, Tulsa, OK).

\section{Results}

Information regarding intra- and inter-day reliability of tissular, metabolic, hydric and ionic indexes is presented in Table $\mathbf{2 - 5}$, respectively. Whatever the measurement, we did not find any systematic bias within or between days.

The relative reliability of tissular indexes was high to very high $(0.77<$ ICC $<0.99)$. However, we found substantial differences in the absolute reliability, since the SEM of fat-free mass was less than
$1.8 \%$, while that of fat mass was higher than $10 \%$ ( $\triangleright$ Table 2 ). This difference inevitably impacted the MD, since the observed difference required to exceed the noise in the measurement during repeated testing was less than $5 \%$ for fat-free mass, whereas it ranged from 27.7 to $41.5 \%$ for fat mass.

The relative reliability of metabolic indexes was high to very high $(0.71<$ ICC $<0.95)$, whereas absolute reliability ranged from 2.7 to $7 \%$. With the exception of the active cellular mass protein content, whose morning SEM and MD were more than twice that of the afternoon ( $\triangleright$ Table 3), absolute reliability could be considered as homogeneous across indexes or time of the day.

With the exception of intracellular water and the ratio between extracellular and intracellular water during the afternoon (ICC <0.67), all hydric indexes were associated with a high to very high relative reliability $(0.79<I C C<0.98$; $>$ Table 4$)$. The absolute reliability of all measures, including intracellular water and the ratio between extracellular and intracellular water, could be considered as very reasonable (SEM $<4 \%$ and $M D<11 \%$ ).

Information regarding ionic indexes is presented in $>$ Table $\mathbf{5 .}$ With the exception of extracellular sodium, particularly in the afternoon, all ionic indexes could be considered as very highly reliable because their ICC ranged from 0.91 to 0.99 . Absolute reliability appeared to be better in the morning (SEM $<2.5 \%$ and $\mathrm{MD}<6.9 \%$ ) in comparison with the afternoon (SEM $<4.5 \%$ and $\mathrm{MD}<12.5 \%$ )

\section{Discussion}

The purpose of this study was to examine the intra- and inter-day reliability of body composition measurements provided by a multifrequency bioelectrical impedance meter, the Z-Metrix (BioparHom, Bourget du Lac, France). Our three main results were 1) the absence of systematic bias within and between days; 2) a high to very high relative reliability of measurements; and 3) an absolute reliability that vary substantially according to the measurements or the time of day.

Whatever the dimension of physical fitness or performance, the cornerstone of an athlete's follow-up is the appreciation of change in order to guide the decision-making of coaches. Change can be defined as a valid confirmation of improvement or worsening in a measurement over a given time span due to intervention [6]. Reliability is a key feature in the tracking of change, because small changes are easier to detect when the reliability is high. The assessment of reliability relies on a specific assessment of three components: systematic bias, relative reliability and absolute reliability [1]. The absence of systematic bias and the high to very high relative reliability of the body composition measurements provided by bioelectrical impedance meters have been consistently reported in the literature $[7,12,20]$. Results obtained with the Z-Metrix are in agreement with the literature and confirm all the interest in bioelectrical impedancemetry to assess body composition. It does not mean, however, that these measurements would be useful in the follow-up of athletes, because bias and relative reliability do not give information about changes at the individual level. The key statistic for this purpose is absolute reliability, and more precisely, the minimum difference considered as real, which represents the limit under which the observed difference is within what we might expect to see in repeated testing just due to measurement noise [21]. 
- Table 2 Intra- and inter-day reliability of tissular indexes.

\begin{tabular}{|c|c|c|c|c|c|c|c|}
\hline Parameter & Day 1 mean \pm SD & Day 2 mean \pm SD & Day 3 mean $\pm S D$ & Day 4 mean $\pm S D$ & ICC & SEM \% & MD \% \\
\hline \multicolumn{8}{|l|}{ Morning } \\
\hline FM (\%) & $14.3 \pm 5.6$ & $13.7 \pm 5.7$ & $13.8 \pm 5.7$ & $13.1 \pm 6.1$ & 0.86 & 15.0 & 41.5 \\
\hline FFM (\%) & $44.2 \pm 7.3$ & $44.0 \pm 7.1$ & $44.2 \pm 7.4$ & $44.3 \pm 7.1$ & 0.99 & 1.5 & 4.2 \\
\hline BMC (\%) & $3.3 \pm 0.4$ & $3.4 \pm 0.4$ & $3.4 \pm 0.4$ & $3.4 \pm 0.4$ & 0.93 & 3.0 & 8.2 \\
\hline \multicolumn{8}{|l|}{ Afternoon } \\
\hline $\mathrm{FM}(\%)$ & $13.4 \pm 5.4$ & $13.5 \pm 6.4$ & $13.3 \pm 5.5$ & $13.1 \pm 5.2$ & 0.95 & 10.0 & 27.7 \\
\hline FFM (\%) & $45.0 \pm 6.7$ & $44.7 \pm 6.5$ & $44.8 \pm 6.8$ & $44.8 \pm 6.8$ & 0.99 & 1.8 & 5.0 \\
\hline BMC (\%) & $3.4 \pm 0.5$ & $3.3 \pm 0.6$ & $3.4 \pm 0.5$ & $3.4 \pm 0.4$ & 0.77 & 6.8 & 18.8 \\
\hline
\end{tabular}

Table 3 Intra- and inter-day reliability of metabolic indexes.

\begin{tabular}{|c|c|c|c|c|c|c|c|}
\hline Parameter & Day 1 mean \pm SD & Day 2 mean \pm SD & Day 3 mean \pm SD & Day 4 mean \pm SD & ICC & SEM \% & MD \% \\
\hline \multicolumn{8}{|l|}{ Morning } \\
\hline MA (a.u.) & $7.2 \pm 0.6$ & $7.2 \pm 0.7$ & $7.1 \pm 0.7$ & $7.2 \pm 0.7$ & 0.78 & 3.9 & 10.8 \\
\hline QCA (\%) & $49.4 \pm 4.7$ & $48.5 \pm 6.4$ & $49.2 \pm 5.2$ & $49.2 \pm 4.8$ & 0.79 & 4.9 & 13.6 \\
\hline $\mathrm{ACM}_{\mathrm{AV}}$ (a.u.) & $0.8 \pm 0.1$ & $0.8 \pm 0.1$ & $0.8 \pm 0.1$ & $0.8 \pm 0.1$ & 0.85 & 4.0 & 10.9 \\
\hline $\mathrm{ACM}_{\mathrm{PC}}(\%)$ & $13.4 \pm 1,8$ & $13.2 \pm 1.8$ & $13.4 \pm 1.8$ & $13.4 \pm 1.7$ & 0.72 & 7.0 & 19.3 \\
\hline \multicolumn{8}{|l|}{ Afternoon } \\
\hline MA (a.u.) & $7.4 \pm 0.6$ & $7.2 \pm 0.5$ & $7.1 \pm 0.7$ & $7.1 \pm 0.7$ & 0.74 & 4.2 & 10.8 \\
\hline QCA (\%) & $50.2 \pm 4.4$ & $49.3 \pm 4.8$ & $49.1 \pm 4.9$ & $49.1 \pm 5.3$ & 0.91 & 2.7 & 7.6 \\
\hline $\mathrm{ACM}_{\mathrm{DI}}$ (a.u.) & $0.7 \pm 0.1$ & $0.8 \pm 0.1$ & $0.8 \pm 0.1$ & $0.8 \pm 0.1$ & 0.71 & 5.3 & 14.7 \\
\hline $\mathrm{ACM}_{\mathrm{PC}}(\%)$ & $13.5 \pm 1.7$ & $13.5 \pm 1.7$ & $13.3 \pm 1.7$ & $13.4 \pm 1.7$ & 0.95 & 2.9 & 8.1 \\
\hline \multicolumn{8}{|c|}{ 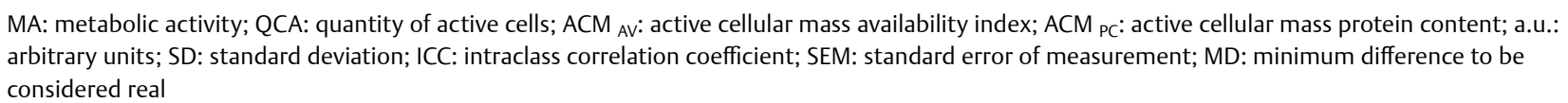 } \\
\hline
\end{tabular}

- Table 4 Intra- and inter-day reliability of hydric indexes.

\begin{tabular}{|c|c|c|c|c|c|c|c|}
\hline Parameter & Day 1 mean \pm SD & Day 2 mean $\pm S D$ & Day 3 mean \pm SD & Day 4 mean \pm SD & ICC & SEM \% & MD \% \\
\hline \multicolumn{8}{|l|}{ Morning } \\
\hline TW (L) & $44.9 \pm 8.4$ & $44.4 \pm 7.5$ & $45.3 \pm 7.9$ & $44.8 \pm 7.6$ & 0.98 & 1.9 & 5.3 \\
\hline EW (L) & $19.1 \pm 3.5$ & $18.9 \pm 3.1$ & $19.4 \pm 3.2$ & $19.1 \pm 3.0$ & 0.97 & 2.4 & 6.5 \\
\hline IW (L) & $26.9 \pm 5.1$ & $26.7 \pm 4.9$ & $26.9 \pm 5.3$ & $27.0 \pm 5.2$ & 0.98 & 2.4 & 6.6 \\
\hline EW/IW (\%) & $71.5 \pm 4.6$ & $71.4 \pm 4.8$ & $72.5 \pm 5.2$ & $71.7 \pm 5.1$ & 0.79 & 3.0 & 8.4 \\
\hline LBMH (\%) & $68.5 \pm 6.3$ & $67.5 \pm 6.1$ & $68.8 \pm 5.7$ & $68.2 \pm 5.1$ & 0.92 & 2.2 & 6.2 \\
\hline \multicolumn{8}{|l|}{ Afternoon } \\
\hline TW (L) & $45.5 \pm 8.2$ & $45.7 \pm 7.5$ & $46.5 \pm 7.5$ & $46.1 \pm 7.5$ & 0.97 & 2.9 & 8.1 \\
\hline EW (L) & $19.2 \pm 3.4$ & $19.4 \pm 3.1$ & $19.8 \pm 3.1$ & $19.6 \pm 3.0$ & 0.95 & 3.6 & 10.0 \\
\hline IW (L) & $28.0 \pm 5.0$ & $27.7 \pm 4.8$ & $27.8 \pm 4.8$ & $27.7 \pm 5.0$ & 0.61 & 2.8 & 7.8 \\
\hline EW/IW (\%) & $68.6 \pm 4.9$ & $70.2 \pm 4.7$ & $71.6 \pm 6.0$ & $71.2 \pm 5.1$ & 0.67 & 4.0 & 11.2 \\
\hline LBMH (\%) & $67.1 \pm 5.9$ & $68.1 \pm 6.4$ & $68.1 \pm 5.1$ & $68.3 \pm 6.1$ & 0.86 & 3.3 & 9.1 \\
\hline
\end{tabular}

TW: total water; EW: extracellular water; IW: intracellular water; LBMH: lean body mass hydration; SD: standard deviation; ICC: intraclass correlation coefficient; SEM: standard error of measurement; MD: minimum difference to be considered real

This noise, or error of measurement, can be related to the device itself (mechanical or electrical drift), to the operator, to the athlete, to the protocol, and to environmental conditions during the test. In our study, some measurements like body fat were associated with poor absolute reliability because the cut-off value was $>27 \%$, whereas other measurements, like most of the hydric and ionic measurements, had a decreased absolute reliability (i. e. an increased cut-off value) during the afternoon when compared with the morning. The poor absolute reliability of fat mass has already been underscored in the meta-analysis by Talma et al. [20].

Several explanations can be put forward to understand these observations. At the device level, bioelectrical impedance is gen- 
- Table 5 Intra- and inter-day reliability of ionic indexes.

\begin{tabular}{|c|c|c|c|c|c|c|c|}
\hline Parameter & $\begin{array}{c}\text { Day } 1 \\
\text { mean } \pm \text { SD }\end{array}$ & $\begin{array}{c}\text { Day } 2 \\
\text { mean } \pm \text { SD }\end{array}$ & $\begin{array}{c}\text { Day } 3 \\
\text { mean } \pm \text { SD }\end{array}$ & $\begin{array}{c}\text { Day } 4 \\
\text { mean } \pm S D\end{array}$ & ICC & SEM \% & MD \% \\
\hline \multicolumn{8}{|l|}{ Morning } \\
\hline Sodium (mmol) & $3058 \pm 572$ & $3027 \pm 513$ & $3086 \pm 540$ & $3056 \pm 519$ & 0.98 & 1.9 & 5.3 \\
\hline Potassium (mmol) & $3959 \pm 817$ & $3916 \pm 735$ & $4000 \pm 773$ & $3957 \pm 742$ & 0.98 & 2.1 & 5.9 \\
\hline Sodium $_{\mathrm{EC}}\left(\mathrm{mmol}^{\left.\mathrm{l} \mathrm{I}^{-1}\right)}\right.$ & $173 \pm 4$ & $173 \pm 4$ & $173 \pm 3$ & $173 \pm 3$ & 0.75 & 1.0 & 2.7 \\
\hline Potassium $_{\mathrm{EC}}\left(\mathrm{mmol}^{\left.\mathrm{l} \mathrm{I}^{-1}\right)}\right.$ & $9.4 \pm 1.1$ & $9.5 \pm 1.2$ & $9.3 \pm 1.1$ & $9.4 \pm 1.1$ & 0.95 & 2.4 & 6.8 \\
\hline Potassium ${ }_{\mathrm{IC}}\left(\mathrm{mmol}^{\prime} \mathrm{I}^{-1}\right)$ & $128 \pm 14$ & $129 \pm 13$ & $127 \pm 13$ & $129 \pm 15$ & 0.94 & 2.3 & 6.5 \\
\hline Creatinine (g.day ${ }^{-1}$ ) & $1.4 \pm 0.4$ & $1.4 \pm 0.4$ & $1.4 \pm 0.4$ & $1.4 \pm 0.4$ & 0.99 & 1.5 & 4.0 \\
\hline Nitrogen $(\mathrm{g})$ & $1361 \pm 226$ & $1373 \pm 222$ & $1380 \pm 230$ & $1387 \pm 221$ & 0.97 & 2.5 & 6.9 \\
\hline \multicolumn{8}{|l|}{ Afternoon } \\
\hline Sodium (mmol) & $3100 \pm 556$ & $30578 \pm 548$ & $3166 \pm 519$ & $3140 \pm 511$ & 0.93 & 4.5 & 12.5 \\
\hline Potassium (mmol) & $4020 \pm 796$ & $4038 \pm 735$ & $4126 \pm 722$ & $4138 \pm 816$ & 0.94 & 4.4 & 12.2 \\
\hline Sodium $_{\mathrm{EC}}\left(\mathrm{mmol}^{\left.\mathrm{l} \mathrm{I}^{-1}\right)}\right.$ & $175 \pm 4$ & $174 \pm 4$ & $173 \pm 4$ & $173 \pm 3$ & 0.63 & 1.2 & 3.4 \\
\hline Potassium $_{\mathrm{EC}}\left(\mathrm{mmol} . \mathrm{I}^{-1}\right)$ & $9.7 \pm 1.0$ & $9.6 \pm 1.0$ & $9.4 \pm 1.0$ & $9.5 \pm 1.1$ & 0.91 & 3.0 & 8.4 \\
\hline Potassium $_{\mathrm{IC}}\left(\mathrm{mmol} . \mathrm{I}^{-1}\right)$ & $127 \pm 14$ & $128 \pm 14$ & $129 \pm 16$ & $128 \pm 14$ & 0.93 & 3.0 & 8.4 \\
\hline Creatinine (g.day ${ }^{-1}$ ) & $1.4 \pm 0.4$ & $1.4 \pm 0.4$ & $1.4 \pm 0.4$ & $1.4 \pm 0.4$ & 0.99 & 2.1 & 5.9 \\
\hline Nitrogen $(\mathrm{g})$ & $1426 \pm 197$ & $1429 \pm 211$ & $1424 \pm 217$ & $1428 \pm 211$ & 0.94 & 3.6 & 10.0 \\
\hline
\end{tabular}

erally considered as precise, because the standard error of measurement for resistance and reactance is around $1 \%$ [7]. It also requires limited operator training, which is one of its strengths. As previously underscored, the main difficulty resides in converting the raw impedance signal into body composition. This conversion relies on two assumptions: 1 ) the human body is shaped like a perfect cylinder with a uniform length and cross-sectional area, and 2) the relationship between body composition and electrical impedance is uniform within and between individuals [13]. Although these assumptions are only partially true $[10,13]$, one may consider this error to be constant, and as such to affect the accuracy of the measurements but not their reliability. The conversion of the signal of impedance also requires population-specific equations that account at least for body height, body mass, age, and resistance. If the three first parameters are easy to control, resistance has been shown to be very sensitive to a number of factors, one of the most important being hydration [11]. Therefore, all the factors that potentially modify total body water, such as eating, drinking or exercising, also potentially alter total body resistance and the estimation of fat-free mass [10]. Body position, limb position, electrode position and surface size, as well as skin condition are also known to affect the signal of impedance, thereby influencing the estimation of body composition compartments [14]. The list of factors to reduce measurement noise and improve absolute reliability is long. In our study, hydration, and particularly the water content of the food absorbed during the last meal, was the single factor that was difficult to control within a day and that may have contributed to the lower absolute reliability of hydric and ionic indicators in the afternoon. Afternoon visits were scheduled between 4 and 6 PM, at least three hours after lunch. Participants were also asked to empty their bladder before the session. Although it is indispensable, it has to be recognized that this procedure does not warrant precise control of hydration level. Apart from this point, our protocol fulfilled most of the current recommendations [14]. In spite of this high standardization, hydric and ionic indicators were associated with a low absolute reliability. It does not mean that these measurements should not be used in the follow-up of athletes, but simply that the cut-off value to signify that a real change has occurred should be adapted. Data presented in $>$ Table 4, 5 can be used for this purpose.

\section{Practical implications}

Bioelectrical impedance combines several characteristics that are particularly interesting in the follow-up of athletes because it requires limited operator training, is not operator-dependent, is financially affordable, and is fast and easy to implement. The question for sport scientists and coaches is to determine cut-off values to identify when a real change has occurred and whether it justifies decision-making by the coach. Reliability, and more particularly absolute reliability, is particularly important for this purpose. Our data suggest that some indicators like fat mass or other hydric and ionic indicators are associated with an important error of measurement. It requires the staff highly standardize the protocol, with a preference for morning measurements. It also implies the use of specific cut-off values to support a valid interpretation of changes over a time span.

\section{Conclusion}

The purpose of this study was to examine the intra- and inter-day reliability of body composition measurements provided by a multifrequency bioelectrical impedance meter, the Z-Metrix (BioparHom, Bourget du Lac, France). Our three main results were 1) the absence of systematic bias within and between days; 2 ) a high to very high relative reliability of measurements; and 3) an absolute reliability that varies substantially according to the measurements or the time of day. These data underscore the importance of high standardization and also the need to adjust the cut-off value for 
each parameter in order to make sure that a real change has occurred. Future studies should focus on implementing these measures in real-life conditions with different sports or training loads in order to select the most sensitive ones in the follow-up of athletes.

\section{Acknowledgements}

No funding was received for this work from any organization or any other institution

\section{Conflicts of Interest}

The authors have no conflicts of interest that are directly relevant to the content of this manuscript.

\section{References}

[1] Atkinson G, Nevill AM. Statistical methods for assessing measurement error (reliability) in variables relevant to sports medicine. Sports Med 1998; 26: 217-238

[2] Australian Sports Commission. Physiological Tests for Elite Athletes. Champaign (ILL): Human Kinetics; 2000

[3] Bosquet L, Maquet D, Forthomme B, Nowak N, Lehance C, Croisier JL. Effect of the lengthening of the protocol on the reliability of muscle fatigue indicators. Int J Sports Med 2010; 31: 82-88

[4] Currier D. Elements of Research in Physical Therapy. Baltimore: Williams and Wilkins; 1990

[5] Deurenberg P, Yap M, van Staveren WA. Body mass index and percent body fat: A meta analysis among different ethnic groups. Int J Obes Relat Metab Disord 1998; 22: 1164-1171

[6] Dvir Z. Difference, significant difference and clinically meaningful difference: The meaning of change in rehabilitation. J Exerc Rehabil 2015; 11: 67-73

[7] Fornetti WC, Pivarnik JM, Foley JM, Fiechtner JJ. Reliability and validity of body composition measures in female athletes. J Appl Physiol (1985) 1999; 87: 1114-1122

[8] Graves ], Pollock M, Colvin A, Van Loan M, Lohman T. Comparison of different bioelectrical impedance analyzers in the prediction of body composition. Am J Hum Biol 1989; 1: 603-611
[9] Harriss D], Atkinson G. Ethical standards in sport and exercise science research: 2016 update. Int J Sports Med 2015; 36: 1121-1124

[10] Heyward V. Advanced fitness assessment and exercise prescription. Champaign (ILL): Human Kinetics; 2006: 1-425

[11] Jackson AS, Pollock ML, Graves JE, Mahar MT. Reliability and validity of bioelectrical impedance in determining body composition. J Appl Physiol (1985) 1988; 64: 529-534

[12] Kilduff LP, Lewis S, Kingsley MI, Owen NJ, Dietzig RE. Reliability and detecting change following short-term creatine supplementation: Comparison of two-component body composition methods. J Strength Cond Res 2007; 21: 378-384

[13] Kyle UG, Bosaeus I, De Lorenzo AD, Deurenberg P, Elia M, Gomez JM, Heitmann BL, Kent-Smith L, Melchior JC, Pirlich M, Scharfetter H, Schols AM, Pichard C.Composition of the EWG. Bioelectrical impedance analysis-part I: Review of principles and methods. Clin Nutr 2004; 23: 1226-1243

[14] Kyle UG, Bosaeus I, De Lorenzo AD, Deurenberg P, Elia M, Manuel Gomez J, Lilienthal Heitmann B, Kent-Smith L, Melchior JC, Pirlich M, Scharfetter H, MWJS A, Pichard C.Espen Group. Bioelectrical impedance analysis-part II: Utilization in clinical practice. Clin Nutr 2004; 23: 1430-1453

[15] Martin AD, Ross WD, Drinkwater DT, Clarys JP. Prediction of body fat by skinfold caliper: assumptions and cadaver evidence. Int $\mathrm{J}$ Obes 1985; 9 (Suppl 1): 31-39

[16] Micozzi M, Harris T. Age variations in the relation of body mass index to estimates of body fat and muscle mass. Am J Phys Anthropol 1990; 3: $375-379$

[17] Munro B. Statistical methods for health care research. 3rd ed. New York: Lippincott; 1997: 1-444

[18] Roubenoff R, Baumgartner RN, Harris TB, Dallal GE, Hannan MT, Economos CD, Stauber PM, Wilson PW, Kiel DP. Application of bioelectrical impedance analysis to elderly populations. J Gerontol A Biol Sci Med Sci 1997; 52: M129-M136

[19] Smye SW, Sutcliffe J, Pitt E. A comparison of four commercial systems used to measure whole-body electrical impedance. Physiol Meas 1993; 14: $473-478$

[20] Talma H, Chinapaw M], Bakker B, HiraSing RA, Terwee CB, Altenburg TM. Bioelectrical impedance analysis to estimate body composition in children and adolescents: A systematic review and evidence appraisal of validity, responsiveness, reliability and measurement error. Obes Rev 2013; 14: 895-905

[21] Weir JP. Quantifying test-retest reliability using the intraclass correlation coefficient and the SEM. J Strength Cond Res 2005; 19: 231-240 